\title{
Myeloid-derived Suppressor Cell and Non-classical Monocyte Frequencies Are Directly Related to the Immunological Status of Patients With Active Tuberculosis
}

\section{Nicolas Amiano}

Instituto de Química Biológica, Facultad de Ciencias Exactas y Naturales (IQUIBICEN). Universidad de Buenos Aires. UBA - CONICET

\section{Joaquín Pellegrini}

Instituto de Química Biológica, Facultad de Ciencias Exactas y Naturales (IQUIBICEN). Universidad de Buenos Aires. UBA - CONICET

\section{María Morelli}

Instituto de Química Biológica, Facultad de Ciencias Exactas y Naturales (IQUIBICEN). Universidad de Buenos Aires. UBA - CONICET

\section{Agustín Rolandelli}

Instituto de Química Biológica, Facultad de Ciencias Exactas y Naturales (IQUIBICEN). Universidad de Buenos Aires. UBA - CONICET

\section{Camila Martinena}

Instituto de Química Biológica, Facultad de Ciencias Exactas y Naturales (IQUIBICEN). Universidad de Buenos Aires. UBA - CONICET

\section{Florencia Castello}

Instituto de Química Biológica, Facultad de Ciencias Exactas y Naturales (IQUIBICEN). Universidad de Buenos Aires. UBA - CONICET

Nicolás Casco

Hospital Muñiz

Lorena Ciallella

Hospital Muñiz

\section{Graciela de Casado}

Hospital Muñiz

\section{Rita Armitano}

Hospital Piñero

\section{Juan Stupka}

Hospital Piñero

\section{Claudio Gallego}




\section{Juan Palmero}

Hospital Muñiz

\section{Verónica García}

Instituto de Química Biológica, Facultad de Ciencias Exactas y Naturales (IQUIBICEN). Universidad de Buenos Aires. UBA - CONICET

Nancy Tateosian ( $\square$ nantateosian@gmail.com )

Instituto de Química Biológica, Facultad de Ciencias Exactas y Naturales (IQUIBICEN). Universidad de Buenos Aires. UBA - CONICET

\section{Research Article}

Keywords: Active Tuberculosis, Non-classical Monocytes, Myeloid-derived suppressor cells, Anti TBtreatment

Posted Date: January 18th, 2021

DOI: https://doi.org/10.21203/rs.3.rs-145332/v1

License: (c) (i) This work is licensed under a Creative Commons Attribution 4.0 International License. Read Full License 


\section{Abstract}

Alterations of myeloid cell populations have been reported in patients with tuberculosis (TB). Since myeloid-derived suppressor cells (MDSC) and non-classical monocytes $\left(\mathrm{CD} 14^{+} \mathrm{CD} 16^{+}\right)$seem to play an important role in TB, we studied the relationship between these cells and the immunological status of patients. TB patients were classified as high responders (HR-TB) or low responders (LR-TB) according to their T cell responses against a whole cell lysate of Mycobacterium tuberculosis (Mtb-Ag). Thus, LR-TB, individuals with severe disease, display a weaker immune response to $M t b$ compare to HR-TB, subjects with strong immunity against the bacteria. We observed that LR-TB presented higher percentages of $\mathrm{CD} 14^{+} \mathrm{CD} 16^{+}$monocytes as compared to HR-TB and healthy donors (HD). Moreover, monocyte-like (MMDSC) and polymorphonuclear-like MDSC (PMN-MDSC) were increased in TB patients as compared to HD. Furthermore, the proportion of M-MDSC of TB patients inversely correlated with the levels of IFN- $y$ released after $M t b-A g$ stimulation. We also found that LR-TB displayed the highest levels of circulating MMDSC. Interestingly, in LR-TB, the frequencies of non-classical monocytes and M-MDSC were restored after only three weeks of anti-TB treatment. Together, our findings show a direct relationship between the immunological status of TB patients and the levels of different circulating myeloid cell populations.

\section{Introduction}

Tuberculosis (TB) is a communicable disease caused by the bacterial pathogen Mycobacterium tuberculosis (Mtb), which together with SARS-CoV-2 in $2020^{1}$, is the leading cause of death from a single infectious agent ${ }^{2}$. Globally, an estimated 10.0 million people fell ill with TB and there were 1.2 million died because of this disease in 2019². Despite the use of the bacilli Calmette-Guérin (BCG) vaccine and the current chemotherapeutic treatments, TB remains as one of the top 10 causes of death worldwide ${ }^{2}$. Thus, the main challenges in developing new strategies to fight TB are focused on the reduction of the duration and complexity of drug regimens ${ }^{2}$. Therefore, there is a critical need to understand the mechanisms of immune controlto achieve a significant impact on the epidemic ${ }^{3}$.

After infection, Mtb interacts with different cells of both, innate and adaptive immune compartments. These cells play an important role in the modulation and the development ofthe pathology ${ }^{4}$. An efficient host protection against $M t b$ infection is associated with the induction, activation and proliferation of Th1 and Th17 cells ${ }^{5-7}$, whom promote the release of cytokines such as IL-2, TNF- $\alpha$, IFN- $\gamma$, and IL-17; and the activation of effector monocytes ${ }^{5-7}$. Moreover, it has been previously demonstrated that reduced IFN- $\gamma$ production is a marker of severe disease ${ }^{8}$ and deficiencies in the IL-12-IFN-Y-STAT1 signaling pathway lead to the dissemination of mycobacterial infections ${ }^{3,9}$. Nevertheless, how $M$ tb is able to evade host immune surveillance and persist, particularly inside myeloid cells, is not yet fully elucidated. Inflammatory myeloid cells are key players in the pathophysiology of tuberculosis ${ }^{10}$. In fact, it has been proposed that the phenotype of the populations of myeloid cells involved in early granuloma formation may influence substantially the progression of TB and the outcome of the infection $7,11,12$. Many studies have shown that mycobacterial infections can also affect the differentiation of progenitors and immature myeloid 
cells to mature and polarized phagocytes ${ }^{11} . M t b$ can alter the activation of recruitedmacrophages and shape thecytokines and chemokinesthat they produce ${ }^{13}$. Furthermore, we have previously demonstrated that IFN- $\gamma$ and IL17A differentially regulate the autophagy process in Mtb-infected monocytes derived from TB patients in correlation with the severity of the disease ${ }^{5,14}$. Phenotypically and functionally different subsets of monocytes were identified based on the relative expression of CD14 (co-receptor for toll-like receptor 4 ) and CD16 (Fc gamma receptor IIla) ${ }^{15}$. Besides, it has been described that the ability of circulating non-classical monocytes $\left(\mathrm{CD} 14^{+} \mathrm{CD} 16^{+}\right)$to differentiate into dendritic cells and induce T-cell activation is decreased in TB patients ${ }^{16}$.

Myeloid-derived suppressor cells (MDSC), are other relevant population of phagocytes that participate in TB immunopathogenesis ${ }^{17}$. Although little is known about the role of MDSC, accumulating evidence clearly demonstrates their capacity to suppress T-cell responses in TB ${ }^{17}$. Moreover, MDSC have been reported to be linked to disease progression $7,18,19$. MDSC described in TB mainly comprise two different subsets: monocyte-like MDSC (M-MDSC) and polymorphonuclear-like MDSC (PMN-MDSC) cells 20,21. Although no specific markers have been described for MDSC identification ${ }^{17}$, human M-MDSC were shown to express CD14, CD33, and CD11b with a lack of CD15 and low or no HLA-DR expression ${ }^{20-23}$. On the other hand, PMN-MDSC express CD15, CD33 and CD11b inthe absence of CD14 andlow or no HLA-DR expression ${ }^{20-23}$.

The accumulation of MDSC during mycobacterial infections wasfirst reported in mice models 24,25 . Furthermore, the expansion of MDSCs was observed bothin blood of patients with pulmonary TB, and in pleural fluid of extra-pulmonary pleural $\mathrm{TB}^{7}$. Besides, it was also described a clear decrease of circulating MDSC frequencies at the end of successful anti-TB treatments ${ }^{7}$. However, further characterization of these cells considering the immunological status of the individuals is currently needed.

Due to the important role of monocytes and MDSC during Mtb infection, in this study, we evaluated the levels of these circulating myeloid cells in TB patients.

We found a differential expansion profile of monocytes and MDSC accordingto the immunological status of TB patients. We could observe that low responder (LR-TB) TB patients presented higher percentages of circulating $\mathrm{CD} 14^{+} \mathrm{CD} 16^{+}$monocytes and M-MDSC as compared tohigh responder (HR-TB) patientsand healthy donors (HD). Furthermore, levels of $\mathrm{CD} 14^{+} \mathrm{CD} 16^{+}$monocytes and M-MDSC were restored to normal after the first three weeks of anti-TB treatment. Therefore, wecould show a direct relationship between the immunological status of TB patients and the frequencies of circulating CD14 ${ }^{+} \mathrm{CD} 16^{+}$ monocytes and M-MDSC. Taking into account the crucial role of cellular immunity during TB, these findings could be important pieces to better understand $M t b$ infection and disease.

\section{Results}


Healthy BCG-vaccinated donors (HD) and pulmonaryTBpatientswere analyzed. Patients with active disease were classified ashigh responders(HR-TB) or low responders (LR-TB) based on their in vitro lymphocyte responses against $M t b-A g$.HR-TB patients display significant proliferative responses, IFN- $\gamma$ production and increased percentage of signaling lymphocyte activation molecule (SLAMF1) positive cells after the exposure to the antigen. On the other hand, LR-TBexhibit low proliferative responses, IFN- $\gamma$ production and SLAMF1-positive cells ${ }^{26}$. Therefore, LR-TB present a more severe TB disease. Clinical and immunological parameters of participating TB patientsare shown inTable 1.

\section{Analysis of circulating monocyte subsets frequencies in TB patients and healthy donors}

Previous reports have shown that $\mathrm{CD} 16^{+}$monocytes are expanded in TB infection in association with the severity of the disease $\mathrm{e}^{27,28}$. Then, we initially decided to analyze the subsets of circulating blood monocytes in our study population based on the expression of CD14 and CD16 molecules. TB patients showed higher percentage of circulating $\mathrm{CD} 14^{+} \mathrm{CD} 16^{+}$cells than $\mathrm{HD}\left(\% \mathrm{CD} 14^{+} \mathrm{CD} 16^{+}\right.$cells $(\mathrm{TB})=15.4 \pm$ 3.5; \%CD14 ${ }^{+} \mathrm{CD} 16^{+}$cells $(\mathrm{HD})=8.1 \pm 0.9$. Mean $\pm \mathrm{SEM}$. ${ }^{*} P<0.01$, Mann-Whitney $U$ test). Furthermore, the percentage of $\mathrm{CD} 14^{+} \mathrm{CD} 16^{+}$monocytes was also analyzed in the two groups of TB patients classified as previously described. As we described, this classification is based on the immunological status of patients. No perturbation of classical monocytes (CD14 $\left.{ }^{+} \mathrm{CD} 16^{-}\right)$was observed when comparing HR-TB, LR-TB and HD (Fig. 1a), but, surprisingly, we found that LR-TB patients presented the highest percentages of CD $14^{+} \mathrm{CD} 16^{+}$circulating cells (Fig. 1b). On the other hand, HR-TB patients showed significantly lower levels of these non-classical monocytes than LR-TB and similar frequencies as compared to those observed in HD (Fig. 1b). Nevertheless, the analysis of the clinical parameters showed that there were no differences in monocytes count between HR-TB and LR-TB. No differences were also observed in the number of circulating monocytes between patients classified as mild, moderate or severe according to radiological lesions, nor between patients classified according to acid-fast bacilli in sputum smear (see Supplementary Fig. S1 online). In view of our results, and to evaluate the potential use of $C D 14^{+} C D 16^{+}$ cells frequencies to discriminate individuals LR-TB from HR-TB, we performed a ROC analysis (Fig. 1e). From this study, significantresults were obtained (AUC $=0.8750 ; P<0.01 ; 95 \% \mathrm{Cl}: 0.68-1.07$ ), demonstrating that the percentages of circulating non-classical monocytes allow differentiating between these patients with distinct immunological status (Cut-off $=8.31 \%$; Sensitivity $=81.8 \%$; Specificity $=$ $87.5 \%)$.

\section{Analysis of circulating MDSC in TB patients and healthy donors}

Then, we decided to compare the frequencies of circulating MDSC in TB patients and HD. PBMC were obtained by Ficoll-Hypaque gradient and then MDSC percentages were analyzed by flow cytometry. Significant higher levels of monocyte-like MDSC (M-MDSC) and polymorphonuclear-like MDSC (PMNMDSC) were observed in TB patients when compared with HD (Fig. 2a and 2b).

As previous studies have suggested that M-MDSCare more potent immune suppressors than PMNMDSC $^{29}$, we then investigated whether M-MDSC and PMN-MDSC subsets presented any correlation with 
IFN-y levels produced by $T$ cells after Mtb-Ag stimulation. For this, we stimulated PBMC with the whole cell lysateof the bacteria for $48 \mathrm{~h}$ and then, IFN- $\gamma$ levels were measured in culture supernatants. The performed analysis showed that the percentages of M-MDSC were inversely correlated with IFN- $\gamma$ levels in TB patients. However, no correlation between the percentages of PMN-MDSC and the levels of IFN-y were detected (Fig. $\mathbf{2 e}$ and $\mathbf{2 f}$ ). These results suggested an immunosuppressive role of M-MDSC on TB patients' lymphocytes.

To further investigate the clinical significance of MDSC in TB disease, we also studied whether LR-TB, HRTB and HD presented different levels of circulating M-MDSC and PMN-MDSC. The FACS analysis showed significant higher levels of M-MDSC in LR-TB in comparison with HR-TB and HD individuals (Fig. 3a). On the contrary, HR-TB patients presented the highest levels of PMN-MDSC compared to TB-LR and HD (Fig. 3b). Furthermore, significant differences in the production of INF- $y$ and the proliferation of lymphocytes of LR-TB and HR-TB patients were detected after Mtb-Ag stimulation (Fig. $\mathbf{3 c}$ and $\mathbf{3 d}$ ). Correlation analysis showed that the percentage of M-MDSC from HR-TB patients negatively correlated with IFN- $Y$ levels from Mtb-Ag stimulated PBMC. However, there was no correlation between M-MDSC frequencies and IFN-y production by cells from LR-TB patients (Fig. 3e).

In order to evaluate the potential use of circulating M-MDSC frequencies in discriminating LR-TB vs. HRTB, we next also performed a ROC analysis (Fig. 3f) obtaining significant results (AUC $=0.7857 ; P<0.05$; 95\% Cl: 0.58-0.99; Cut-off $=11.0 \%$; Sensitivity $=85.7 \%$; Specificity $=75.0 \%)$. These results demonstrated that, together with the percentages values observed for circulating non-classical monocytes, M-MDSC frequencies could be used as a parameter of disease severity.

\section{Effect of short-term anti-TB treatment on monocytes and MDSC cell frequencies}

It has been previously demonstrated that the levels of monocytes and MDSC are correlated with the TB infection progression ${ }^{7,28,30}$. Furthermore, du Plessis et al. have shown that after a complete and successful anti-TB treatment, MDSC frequencies decrease to the levels observed in healthy controls ${ }^{7}$. Thus, since due to the fact that LR-TB patients present severe TB disease ${ }^{6,26}$, we decided to study whether few weeks of anti-TB treatment affected the frequencies of these circulating cell subsets in these patients. We then compared percentages of blood circulating PMN-MDSC, M-MDSC and classical and non-classical monocytes at the beginning of the anti-TB treatment (blood samples taken during the first week of chemotherapy) and after three weeks (blood samples taken between 14 - 21 days later). No differences in the percentage of classical monocytes (CD14 $\left.{ }^{+} \mathrm{CD} 16^{-}\right)$were observed after 14-21 days of anti-TB treatment. However, we observed a significant decrease in non-classical monocytes $\left(\mathrm{CD} 14^{+} \mathrm{CD} 16^{+}\right)$levels after this short period of treatment (Fig. 4a y 4b). In addition, we analyzed whether the percentages of MDSC subsets were also modified during the first days of chemotherapy. Interestingly, we observed a significant reduction of the frequency of M-MDSC in LR-TB patients (Fig. 4c). In contrast, the percentage of PMN-MDSC was not affected by the anti-TB treatment during this period of time (Fig. 4d). Importantly, IFN-y production and the proliferation index significantly augmented during these three weeks of anti-TB treatment (Fig. 4e and 4f). 
Together, our present findings indicate that non-classical monocytes and M-MDSC would play an important role in TB patients with severe disease. Moreover, the accumulation of these subsets of cells is rapidly reversed after a few days of anti-TB treatment, suggesting that non-classical monocytes and $M$ MDSC could serve as a marker of TB progression or even treatment monitoring.

\section{Discussion}

A spectrum of stages caused by $M t b$ infection leads to identify that some patients can control the bacterial infection and others cannot. The IFN-y producing Th1 cells are essential to control mycobacterial replication ${ }^{31,32}$. Indeed, reduced IFN-y production is a well-known marker of disease severity ${ }^{33}$. In our study population, HR-TB and LR-TB patients were identified based on their T cell responses to $M t b-\mathrm{Ag}^{26}$. In spite of that, Th1 and Th17 cells alone do not explain the resistance/susceptibility to infection and disease ${ }^{6,34}$, suggesting that other actors might be required during the immune regulation of TB. Myeloid cells are a heterogeneous group of cells that plays a major role in the regulation of immune responses in many pathological conditions. However, the imbalance of myeloid cells during human TB has been poorly studied. Therefore, we aimed to investigate the populations of monocyte and MDSC cells during human active TB disease. In the present report, we identify an increase in different circulating myeloid cell populations in severe TB patients. Both, immature myeloid suppressor cells M-MDSC and PMN-MDSC were augmented in blood from TB patients. Furthermore, we found that patients that showing weak or no IFN-y responses to Mtb-Ag presented higher percentages of non-classical $\mathrm{CD} 14^{+} \mathrm{CD} 16^{+}$monocytes as compared to HR-TB and HD (Fig. 1). Moreover, LR-TB individuals presented the highest percentages of circulating M-MDSC.On the contrary, HR-TB individuals displayed the highest percentages of PMN-MDSC (Fig. 3). We further observed that the frequencies of $\mathrm{CD} 14^{+} \mathrm{CD} 16^{+}$monocytes and M-MDSC return to healthy control levels after a short time of anti-TB treatment (Fig. 4). Therefore, we showed that these cell subsets contribute to a better characterization of the immunological profile of TB patients and could be new targets for the development of host-directed therapies.

Previously, we observed that monocytes from LR-TB individuals were unable to activate autophagy process through IL17A at least in part because of a defect in the MAPK1/3 signaling pathway. In contrast, both IFN- $\gamma$ and IL17A increased the levels of autophagy in patients with strong immunity to $M t b^{5}$. Our present results extended those findings, demonstrating that LR-TB patients, individuals whose severity clinical and immunological parameters have been already described ${ }^{6,26}$, showed higher proportions of non-classical monocytes and M-MDSC in peripheral blood as compared to HR-TB patients. In fact, we founded an inverse correlation between IFN- $\gamma$ released in response to $M t b-A g$ stimulation and the levels of circulating M-MDSC. Thus, the proportion of circulating CD14 ${ }^{+} \mathrm{CD} 16^{+}$monocytes and M-MDSC in patients with active TB might be reflecting the severity of the disease.

It was suggested that MDSC are closely correlated with the progression of TB infection in humans ${ }^{7,30}$. In accordance with these reports, we found that MDSC are expanded in peripheral blood of active TB 
patients (Fig. 2). Moreover, we show for the first time that there is a characteristic expansion profile of MMDSC and PMN-MDSC in TB patients related to their immunological status. Our results in PBMC confirmed that the majority of HR-TB patients present augmented percentages of PMN-MDSC. Furthermore, patients with weak immunity to $M t b$ presented the highest levels of circulating M-MDSC. However, an inverse correlation between the percentages of circulating M-MDSC and IFN-y levels was only observed in HR-TB patients (Fig. 3). These would suggest that M-MDSC from HR-TB present more important suppressive effects than M-MDSC from LR-TB. These results are in agreement with those reported previously where a functional correlation between MDSC and TB infection was also suggested ${ }^{7,35}$.Several studies have recently reportedthe function and the accumulation of MDSC in association with pathogen load ${ }^{7,18}$. du Plessis, et al. showed and increased frequency of MDSC during active tuberculosis with suppressed Tcell functions ${ }^{7}$. Furthermore, Knaul, et al. described in a mice model that MDSC are able to phagocyte $M t b$, and released both, pro-inflammatory (IL-6, IL-1a) and immunomodulatory (IL-10) cytokines while retaining their suppressive capacity ${ }^{18}$. Both, HR and LR-TB patients showed similar number of AFB in sputum smear indicating no differences in the bacterial load between them. This could suggest that the immune microenvironment generated during the chronic inflammation of $M t b$ infection might influence in the accumulation and function of monocytes and MDSC.

Additionally, it has been previously shown that pharmacological therapy reduce the accumulationof myeloid cell population $7,28,30$. Sanchez et al. demonstrated that the expression of CD14, HLA-DR and CD36 wasdecreased in monocytes of TB patients ${ }^{28}$; nevertheless, normal expression of these molecules was restored during anti-TB treatment. Furthermore, in TB patients, MDSC population was reduced at the end of anti-TB therapy ${ }^{7,30}$. However, it is important to mention that in our study we could observe for the first time to our knowledge, a restoration in the circulating levels of $\mathrm{CD} 14^{+} \mathrm{CD} 16^{+}$monocytes and $\mathrm{M}$ MDSC after a short term (three weeks) of anti-TB treatment. In this short period of time we did not observe differences in percentages ofcirculating classical monocytes $\left(\mathrm{CD} 14^{+} \mathrm{CD} 16^{-}\right)$or in PMN-MDSC levels. Therefore, considering previous published data and our present results we hypothesize that some myeloid cell populations could serve as a treatment-response marker during TB. In addition, by providing information about the immunological status of the patient, the circulating levels of CD14 ${ }^{+} \mathrm{CD} 16^{+}$ monocytesand M-MDSC would allow to know about the severity of the active disease in infected individuals.

In summary, our findingsshow that accumulation of circulating non-classical monocytes and monocyticlike MDSC during pulmonary tuberculosis infection reveals the host immunological status. Furthermore, we could describe that the anti-TB treatment rapidly restore the normal levels of these cells. The recruit of these expanded myeloid populations in individuals with a weakened immune response could exacerbate the detrimental pro-inflammatory responses in infected lung tissue and the prognosis of tuberculosis.

\section{Methods}


Study Subjects. Patients with TB were diagnosed at Dr. F. Muñiz or P. Piñero Hospitals (Buenos Aires, Argentina) based on clinical and radiological data together with the identification of acid-fast bacilli (AFB) in sputum. First peripheral blood samples were collected before one week of anti-tuberculosis therapy administration and second samples were obtained between 14 and 21 days of anti-TB treatment. All patients had received anti-tuberculosis (anti-TB) regular therapy for drug sensitive $M t b$ strains. Bacillus Calmette-Guerin (BCG) vaccinated healthy control individuals lacking a history of TB (HD) participated in this study.

Peripheral blood was collected in heparinized tubes from each participant after obtaining a written informed consent for the collection of samples and the subsequent analysis. All methods were carried out in accordance with relevant guidelines and regulations.

The protocols conducted in this work were approved by the Ethics Committees of Muñiz and Piñero Hospitals.

Exclusion criteria and classification of patients. The exclusion criteria were carried out as previously described ${ }^{36}$. Briefly, individuals participating of the study were $18-60$ years old and had no history of diseases affecting the immune system, such as HIV infection, treatment with immunosuppressive drugs,a recent diagnosis of cancer, hepatic or renal disease, pregnancy, or positive serology for other viral (e.g., hepatitis A, B or C), or bacterial (e.g., leprosy, syphilis) infections. Subjects with anticoagulant medication orbleeding disorders that might be at an increased risk of bleeding during the procedure of obtaining the sample were excluded from the study.

Individuals with latent infection were excluded from the present study by using the QuantiFERON-TB Gold Plus kit (Qiagen, Germany, USA).

TB Patients were classified as high responders (HR-TB) or low responders (LR-TB), based on their in vitro lymphocyte responses to a whole cell lysate of $M$. tuberculosis (Mtb-Ag) as previously described ${ }^{26}$. Briefly, HR-TB patients are individuals displaying significant proliferative responses, IFN-y production and an increased percentage of SLAMF1 positive cells after Mtb-Ag stimulation; whereas LR-TB patients exhibit low proliferative responses, IFN- $\gamma$ release and SLAMF1 positive cells. LR-TB patients had more severe pulmonary disease compared with HR individuals.

In the studied population, no differences regarding age distribution or sex were found (Table 2).

Antigen. In vitro stimulation of peripheral blood mononuclear cells (PBMC) was performed with a cell lysate from the virulent Mycobacterium tuberculosis strain H37Rv, prepared by probe sonication (Mtb-Ag) (BEI Resources, NIAID, NIH: Mycobacterium tuberculosis, Strain H37Rv, Whole Cell Lysate, NR-14822).

Cell preparation and reagents. PBMC were isolated by centrifugation on Ficoll-Hypaque (Amersham Biosciences, NJ, USA). Flow cytometry assays were performed after PBMC were washed with PBS plus $1 \% \mathrm{BSA}$ and $0.1 \% \mathrm{NaN}_{3}$ and resuspended in staining buffer (PBS plus $1 \%$ Fetal Bovine Serum (FBS)). 
Viability was determined by exclusion of trypan blue $(\geq 98 \%)$. PBMC were also cultured $\left(1 \times 10^{6}\right.$ cells/mL), with or without $M t b-A g(10 \mu \mathrm{g} / \mathrm{mL})$ with RPMI 1640 medium (Gibco, MD, USA) supplemented with 1\% L-glutamine, 1\% penicillin/streptomycin, and 10\% FBS (Gibco, MD, USA) during $48 \mathrm{~h}$. Then, levels of IFN- $\gamma$ were measured using a commercial ELISA kit (Human IFN- $y$ ELISA MAX Standard Kit, BioLegend, USA) following the manufacturer's instructions.

Flow cytometry. PBMC from TB patients and HD were stained with specific fluorophore-marked antibodies against CD14 (FITC, clone HCD14, BioLegend, USA) and CD16 (APC, clone 3G8, BioLegend, USA) for differentiating monocyte subsets. MDSC classification was performed staining PBMC with specific fluorophore-marked antibodies against CD14 (FITC, clone HCD14, BioLegend, USA), CD11b (PE, clone ICRF44, BioLegend, USA), CD15 (PE/Cy7, clone HI98, BioLegend, USA), and HLA-DR (APC, clone L243, BioLegend, USA). Negative control samples were incubated with irrelevant isotype matched monoclonal antibody (BioLegend, USA).

Gating strategies are shown in the representative density plots of figures.

Cells were incubated for 30 min with the specific antibodies at room temperature in the dark and two washes were made with washing buffer. Samples were analyzed on a FACSAria II flow cytometer (BD Biosciences, CA, USA).

Proliferation index. PBMC were stimulated with $M t b-A g$ for five days and cells were pulsed with $\left[{ }^{3} \mathrm{H}\right] \mathrm{TdR}(1$ $\mu \mathrm{Ci} /$ well) and harvested 16 hours later. $\left[{ }^{3} \mathrm{H}\right] \mathrm{TdR}$ incorporation was measured in a liquid scintillation counter as counts per minute (c.p.m). Proliferation index for each individual was calculated as (c.p.m. after $M t b-A g$ stimulation)/(c.p.m. after culturing with medium).

Statistical analysis. Analysis of variance (ANOVA) and post hoc Tukey's multiple comparisons test were used as indicated in figure legends. The Mann-Whitney $U$ test was used to analyze differences between groups. For categorical variables, the Chi-square (and Fisher's exact) test for homogeneity was performed to compare proportions of subjects between groups. In the graphs each symbol represents an individual and the horizontal lines indicate the mean \pm standard error of the mean (SEM). Correlations were calculated using the non-parametric Spearman correlation test. Receiver operating characteristic (ROC) curve analysis was performed to analyze the predictive value of the frequencies of $\mathrm{CD} 14^{+} \mathrm{CD} 16^{+}$and $\mathrm{M}$ MDSC cells populations, calculating the area under the curve (AUC) and the $95 \%$ confidence interval (Cl). Analyses were performed using GraphPad Prism 8.0.2 software. $P<0.05$ was considered statistically significant.

\section{Declarations}

\section{Acknowledgments}

We thank to Guillermo Piazza, AgustinaErschen and César Salomone for their expert technical assistance. This work was supported by grants from Agencia Nacional de PromociónCientífica y 
Tecnológica (ANPCyT) [PICT2017-1158 and PICT2014-1709 to N.L.T, PICT 2017-1451 to V.E.G. and PICT Start Up 2016-0022 to N.O.A] and Universidad de Buenos Aires (UBACyT) [20020170100127BA to V.E.G. and 200201150200107BA to N.O.A.].

\section{Author contributions}

N.L.T. designed the study. N.O.A., N.L.T., J.M.P., M.P.M., A.R. and F.A.C. were responsible for performing flow cytometry analysis, ELISA and proliferation assays. N.O.A. was in charge of performing the QFT test in $\mathrm{HD}$ to evaluate latent infection. N.O.A., N.L.T., J.M.P., M.P.M. and V.E.G did the data management and analysis. N.L.T., N.O.A., J.M.P. and M.P.M prepared all the figures and tables. J.M.P., M.P.M., A.R., C.M. and F.A.C. were responsible for processing samples and contributed with standard laboratory work. N.O.A. and N.L.T. wrote the manuscript. V.E.G. provided also expert advice. N.C., L.M.C., G.C.C., R.A., J.S., C.G. and J.D.P. were in charge of patient recruitment, diagnosis ofactive tuberculosis and sample collection.Allauthors contributed to data gathering and interpretation, and revision of the report.

\section{Competing interests}

The authors declare no competing interests.

\section{References}

1. World Health Organization. WHO coronavirus disease (COVID-19) dashboard.

2. WHO | Global tuberculosis report 2019.https://www.who.int/tb/publications/global_report/en/.

3. Scriba, T. J. et al. Distinct, specific IL-17- and IL-22-producing CD4+ T cell subsets contribute to the human anti-mycobacterial immune response. J. Immunol. Baltim. Md 1950180, 1962-1970 (2008).

4. Bussi, C. \& Gutierrez, M. G. Mycobacterium tuberculosis infection of host cells in space and time. FEMS Microbiol. Rev.43, 341-361 (2019).

5. Tateosian, N. L. et al. IL17A augments autophagy in Mycobacterium tuberculosis-infected monocytes from patients with active tuberculosis in association with the severity of the disease. Autophagy13, 1191-1204 (2017).

6. Jurado, J. O. et al. IL-17 and IFN-y expression in lymphocytes from patients with active tuberculosis correlates with the severity of the disease. J. Leukoc. Biol.91, 991-1002 (2012).

7. du Plessis, N. et al. Increased frequency of myeloid-derived suppressor cells during active tuberculosis and after recent mycobacterium tuberculosis infection suppresses T-cell function. Am. J. Respir. Crit. Care Med.188, 724-732 (2013).

8. Jh, G. et al. Interleukin-10 downregulates Mycobacterium tuberculosis-induced Th1 responses and CTLA-4 expression. Infection and immunity vol. 64 https://pubmed.ncbi.nlm.nih.gov/8641800/ (1996).

9. Flynn, J. L. Lessons from experimental Mycobacterium tuberculosis infections. Microbes Infect.8, 1179-1188 (2006). 
10. Dorhoi, A. \& Kaufmann, S. H. E. Versatile myeloid cell subsets contribute to tuberculosis-associated inflammation. Eur. J. Immunol.45, 2191-2202 (2015).

11. Khan, A., Singh, V. K., Hunter, R. L. \&Jagannath, C. Macrophage heterogeneity and plasticity in tuberculosis. J. Leukoc. Biol.106, 275-282 (2019).

12. Guirado, E., Schlesinger, L. S. \& Kaplan, G. Macrophages in tuberculosis: friend or foe. Semin. Immunopathol.35, 563-583 (2013).

13. Hickman, S. P., Chan, J. \&Salgame, P. Mycobacterium tuberculosis Induces Differential Cytokine Production from Dendritic Cells and Macrophages with Divergent Effects on Naive T Cell Polarization. J. Immunol.168, 4636-4642 (2002).

14. Rovetta, A. I. et al. IFNG-mediated immune responses enhance autophagy against Mycobacterium tuberculosis antigens in patients with active tuberculosis. Autophagy10, 2109-2121 (2014).

15. Ziegler-Heitbrock, L. et al. Nomenclature of monocytes and dendritic cells in blood. Blood116, e74e80 (2010).

16. Balboa, L. et al. Paradoxical role of CD16+CCR2+CCR5+ monocytes in tuberculosis: efficient APC in pleural effusion but also mark disease severity in blood. J. Leukoc. Biol.90, 69-75 (2011).

17. Magcwebeba, T., Dorhoi, A. \& du Plessis, N. The Emerging Role of Myeloid-Derived Suppressor Cells in Tuberculosis. Front. Immunol.10, (2019).

18. Knaul, J. K. et al. Lung-residing myeloid-derived suppressors display dual functionality in murine pulmonary tuberculosis. Am. J. Respir. Crit. Care Med.190, 1053-1066 (2014).

19. Tsiganov, E. N. et al. Gr-1dimCD11b+ immature myeloid-derived suppressor cells but not neutrophils are markers of lethal tuberculosis infection in mice. J. Immunol. Baltim. Md 1950192, 4718-4727 (2014).

20. Kotzé, L. A. et al. Mycobacterium tuberculosis and myeloid-derived suppressor cells: Insights into caveolin rich lipid rafts. EBioMedicine53, 102670 (2020).

21. Bronte, V. et al. Recommendations for myeloid-derived suppressor cell nomenclature and characterization standards. Nat. Commun.7, 12150 (2016).

22. Cassetta, L. et al. Deciphering myeloid-derived suppressor cells: isolation and markers in humans, mice and non-human primates. Cancer Immunol. Immunother. CI/68, 687-697 (2019).

23. Damuzzo, V. et al. Complexity and challenges in defining myeloid-derived suppressor cells. Cytometry B Clin. Cytom.88, 77-91 (2015).

24. Dietlin, T. A. et al. Mycobacteria-induced Gr-1+ subsets from distinct myeloid lineages have opposite effects on T cell expansion. J. Leukoc. Biol.81, 1205-1212 (2007).

25. Martino, A. et al. Mycobacterium bovis bacillus Calmette-Guérin vaccination mobilizes innate myeloid-derived suppressor cells restraining in vivo T cell priming via IL-1R-dependent nitric oxide production. J. Immunol. Baltim. Md 1950184, 2038-2047 (2010).

26. Pasquinelli, V. et al. Expression of Signaling Lymphocytic Activation Molecule- Associated Protein Interrupts IFN-y Production in Human Tuberculosis. J. Immunol.172, 1177-1185 (2004). 
27. Castaño, D., García, L. F. \& Rojas, M. Increased frequency and cell death of CD16+ monocytes with Mycobacterium tuberculosis infection. Tuberc. Edinb. Scotl.91, 348-360 (2011).

28. Sánchez, M. D. et al. Functional and phenotypic changes in monocytes from patients with tuberculosis are reversed with treatment. Microbes Infect.8, 2492-2500 (2006).

29. Movahedi, K. et al. Identification of discrete tumor-induced myeloid-derived suppressor cell subpopulations with distinct T cell-suppressive activity. Blood111, 4233-4244 (2008).

30. Zhan, X. et al. IFN-Y decreased the suppressive function of CD33+HLA-DRlow myeloid cells through down-regulation of PD-1/PD-L2 signaling pathway. Mol. Immunol.94, 107-120 (2018).

31. Serbina, N. V., Lazarevic, V. \& Flynn, J. L. CD4(+) T cells are required for the development of cytotoxic CD8(+) T cells during Mycobacterium tuberculosis infection. J. Immunol. Baltim. Md 1950167, 6991-7000 (2001).

32. Salgame, P. Host innate and Th1 responses and the bacterial factors that control Mycobacterium tuberculosis infection. Curr. Opin. Immunol.17, 374-380 (2005).

33. Gong, J. H. et al. Interleukin-10 downregulates Mycobacterium tuberculosis-induced Th1 responses and CTLA-4 expression. Infect. Immun.64, 913-918 (1996).

34. Forbes, E. K. et al. Multifunctional, high-level cytokine-producing Th1 cells in the lung, but not spleen, correlate with protection against Mycobacterium tuberculosis aerosol challenge in mice. J. Immunol. Baltim. Md 1950181, 4955-4964 (2008).

35. Lee, J.-M. et al. The restoration of myeloid-derived suppressor cells as functional antigen-presenting cells by NKT cell help and all-trans-retinoic acid treatment. Int. J. Cancer131, 741-751 (2012).

36. Pellegrini, J. M. et al. Neutrophil autophagy during human active tuberculosis is modulated by SLAMF1. Autophagy0, 1-10 (2020).

\section{Tables}

Table 1. Immunological and clinical parameters of TB patients. LR-TB and HR-TB were classified according to IFN- $\gamma$ production [fold-stimulation: $(\mathrm{pg} / \mathrm{mL}$ after $M$ tb-Ag stimulation)/ $(\mathrm{pg} / \mathrm{mL}$ after culturing with medium)]; proliferation [proliferation index: (c.p.m. after Mtb-Ag stimulation)/ (c.p.m. after culturing with medium)]; and increase in the percentage of SLAM-positive cells in response to $M t b-A g$ stimulation. Proportions of lymphocytes, monocytes and neutrophils are shown as percentages of total white blood cells. Time of disease evolution (days) was established by analyzing the following clinical symptoms in patients previous to hospital admission: weight loss, night sweats, symptoms of malaise or weakness, persistent fever, presence of cough, history of shortness of breath, and/or hemoptysis. Mean \pm SEM are shown for continuous data. Categorical data (AFB in sputum smear) are expressed as percentages. ${ }^{\text {a }} P$ values were calculated by the Mann-Whitney $U$ test. ${ }^{b} P$ value was calculated by Chi-Square (and Fisher's exact) test for categorical variables. $P$ values $<0.05$ were considered significant. LR TB: Low responder tuberculosis patients; HR TB: High responder tuberculosis patients. AFB: Acid-Fast Baclli. 


\begin{tabular}{|c|c|c|c|}
\hline Immunological and Clinical Parameters & LR TB & HR TB & $P$-value \\
\hline IFN-ץ production (fold-stimulation) & $44.0 \pm 8.4$ & $154.6 \pm 53.7$ & $0.001^{\mathrm{a}}$ \\
\hline $\begin{array}{l}\text { Proliferative responses } \\
\text { (proliferation index) }\end{array}$ & $1.9 \pm 0.4$ & $6.9 \pm 1.7$ & $0.002^{\mathrm{a}}$ \\
\hline Increase in the \% of SLAM-positive cells & $3.8 \pm 4.8$ & $18.3 \pm 3.4$ & $0.003^{\mathrm{a}}$ \\
\hline Lymphocytes (\%) & $14.6 \pm 4.7$ & $16.9 \pm 1.6$ & $0.661^{\mathrm{a}}$ \\
\hline Monocytes (\%) & $8.3 \pm 1.06$ & $9.8 \pm 1.3$ & $0.397^{a}$ \\
\hline Neutrophils (\%) & $61.9 \pm 7.5$ & $62.2 \pm 6.3$ & $0.977^{\mathrm{a}}$ \\
\hline Time of Disease Evolution (days) & $70.3 \pm 14.2$ & $51.4 \pm 8.1$ & $0.252^{\mathrm{a}}$ \\
\hline AFB in sputum smear (\%) & 94.8 & 93.3 & $0.999^{b}$ \\
\hline$(-)$ & 5.2 & 6.7 & \\
\hline
\end{tabular}

Table 2.Demographic characteristics of healthy donors and tuberculosis patients populations. Age value is expressed as mean \pm SEM. Sex is expressed in percentages. ${ }^{a} P$ value was calculated by ANOVA test; ${ }^{\mathrm{b}} P$ value was calculated by Chi-Square (and Fisher's exact) test for categorical variables. $P$ values $<0.05$ were considered significant. HD: healthy donors; LR TB: Low responder tuberculosis patients and HR TB: High responder tuberculosis patients.

\begin{tabular}{|llllll|}
\hline & & HD & LR TB & HR TB & $P$-value \\
\hline N & 19 & 19 & 15 & - \\
\hline Age & & $29.6 \pm 1.1$ & $26.7 \pm 1.3$ & $25.3 \pm 2.5$ & $0.1031^{\mathrm{a}}$ \\
\cline { 1 - 4 } Sex & Male & 51.6 & 55.6 & 53.4 & \multirow{2}{*}{$0.9406^{\mathrm{b}}$} \\
\cline { 2 - 5 } & Female & 48.4 & 44.4 & 46.6 & \\
\cline { 2 - 5 } & & & & \multicolumn{2}{c}{} \\
\hline
\end{tabular}

\section{Figures}





c.

d.

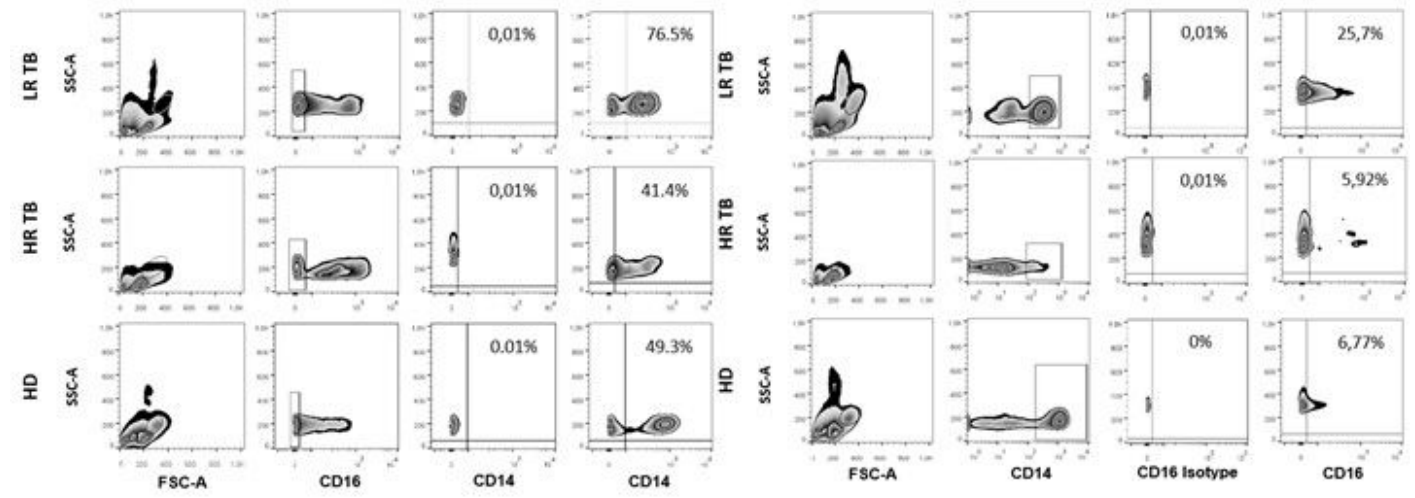

e.

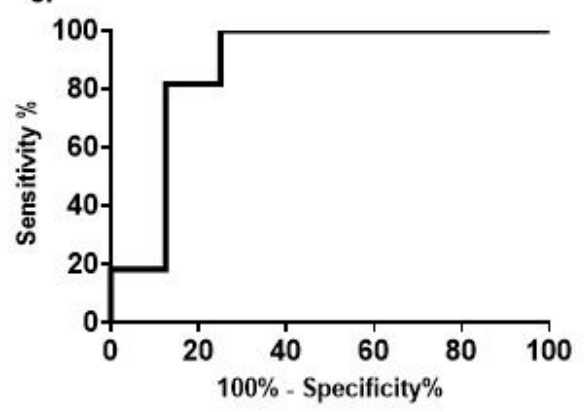

Figure 1 

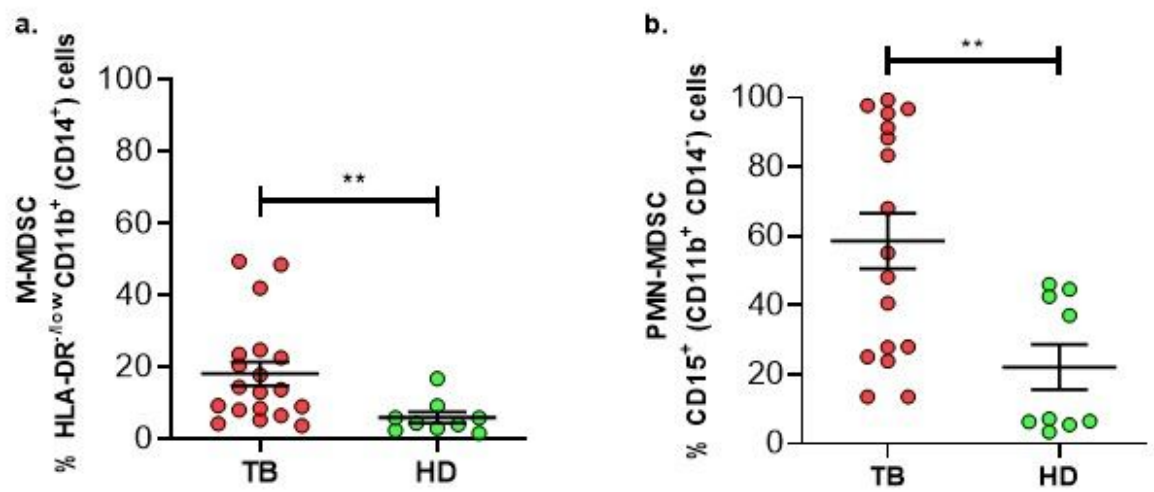

c.
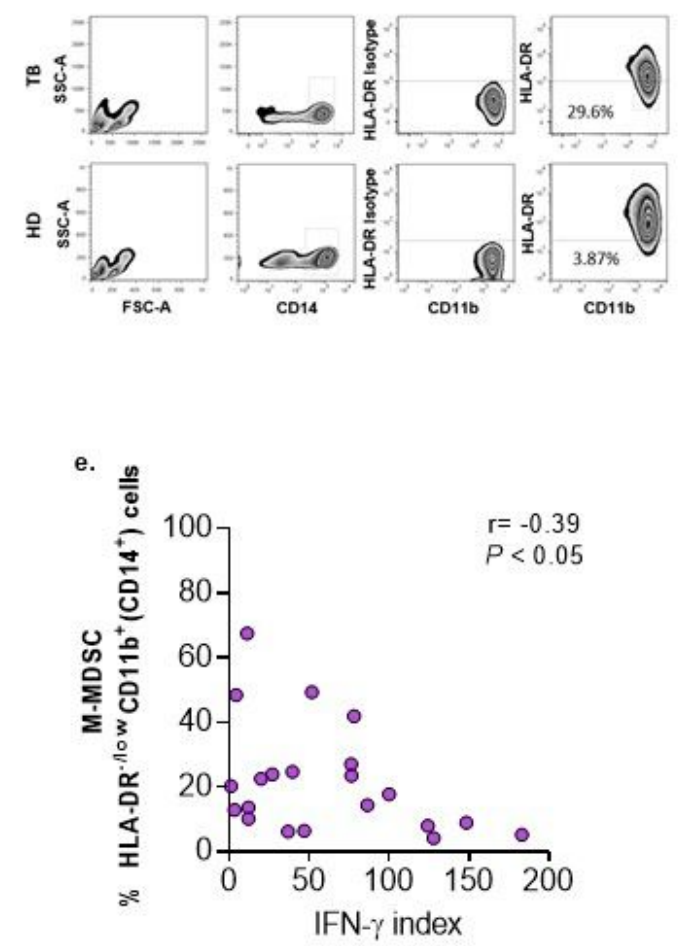

d.
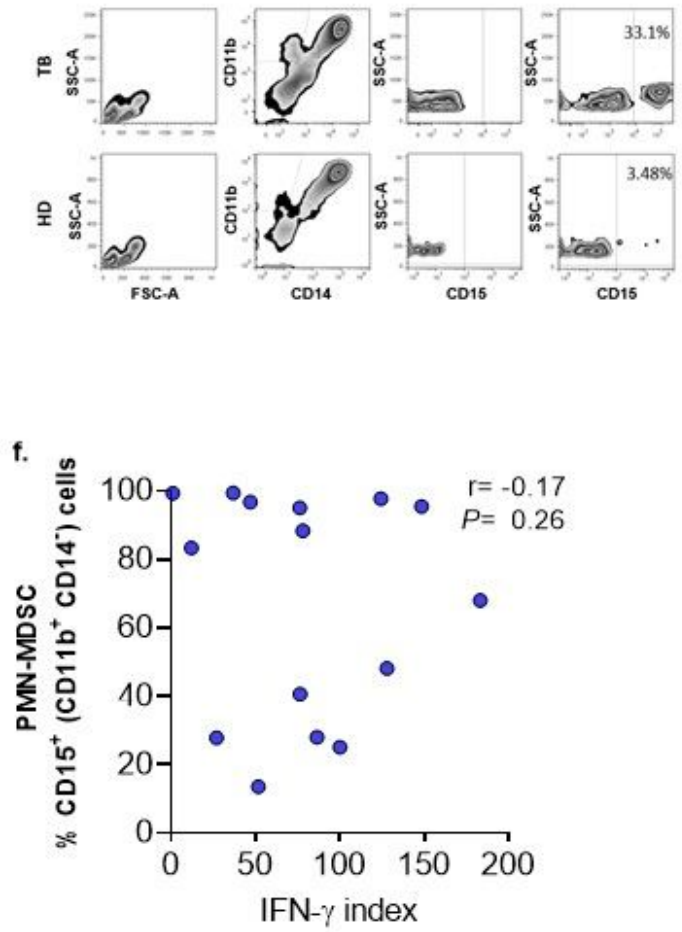

Figure 2 


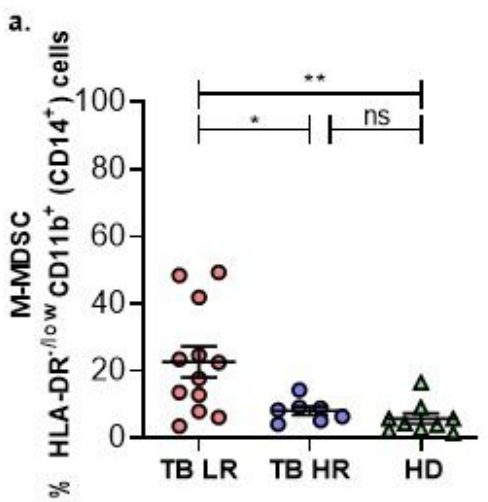

b.
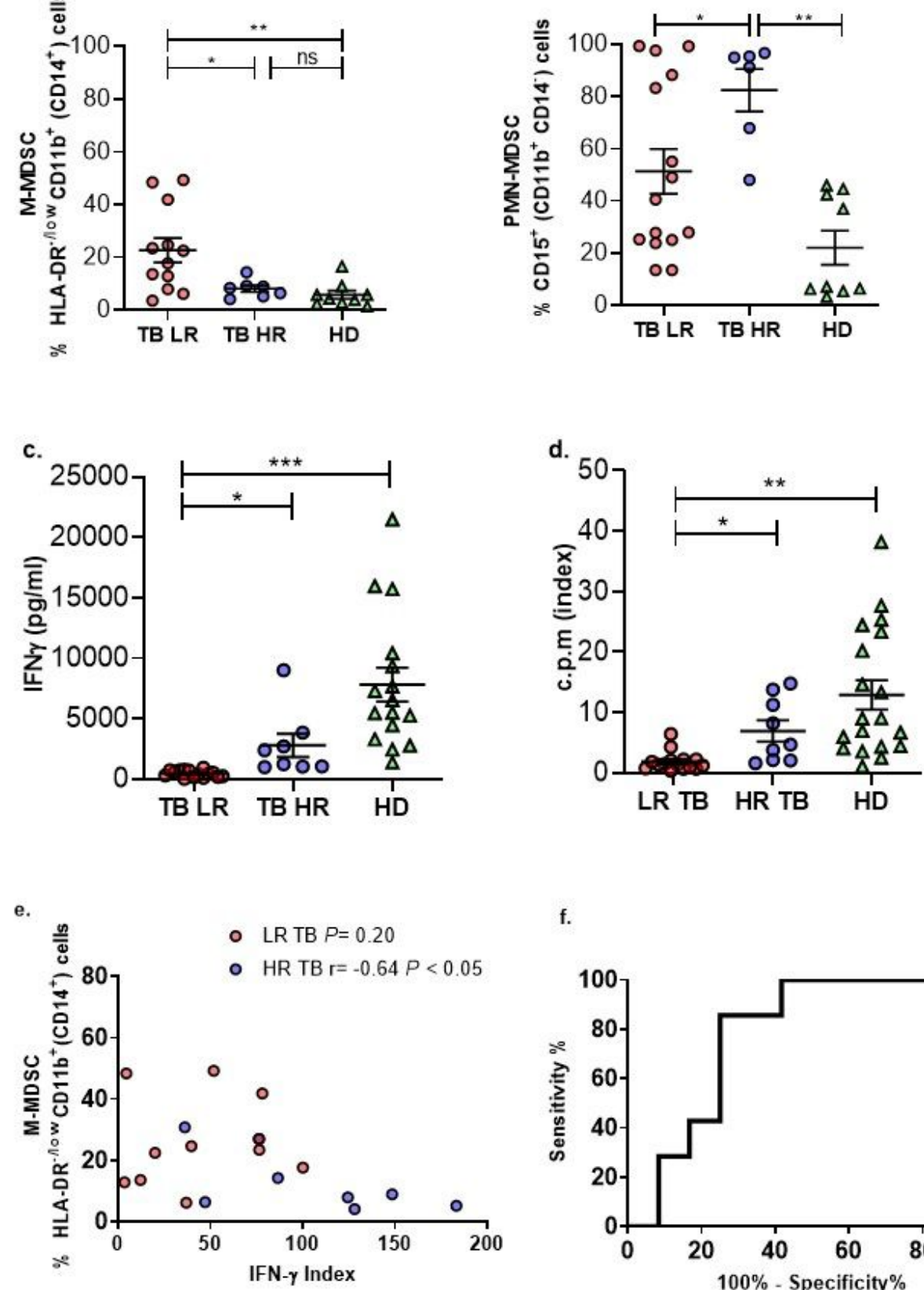

f.



Figure 3 

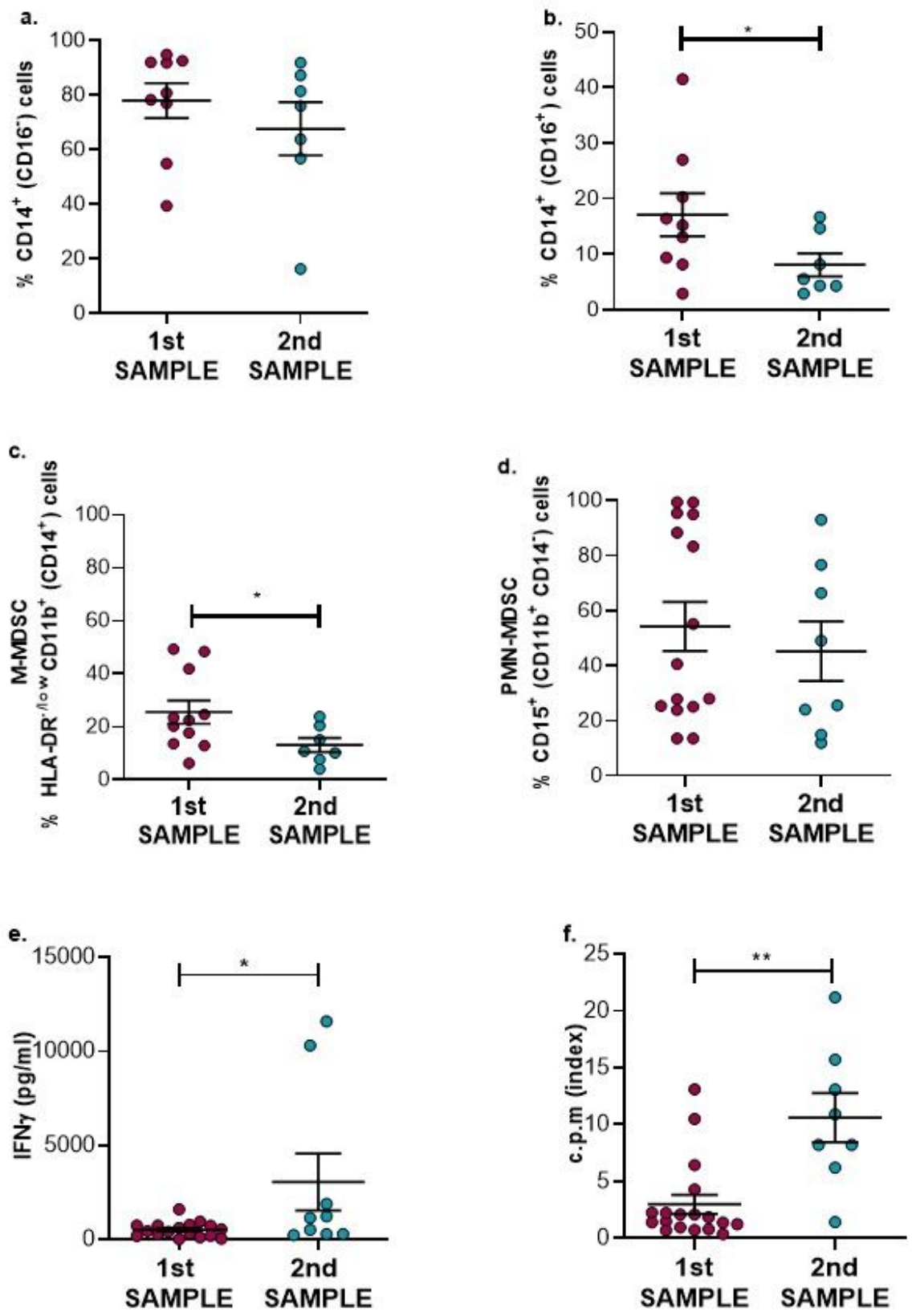

Figure 4

\section{Supplementary Files}

This is a list of supplementary files associated with this preprint. Click to download.

- SupplementaryFigureS1.pdf 